errors of metabolism currently depends on sophisticated biochemical analysis. NMR analysis can simultaneously analyze the mixture of compounds in biological fluids, but complete analysis of biological fluids by NMR will not be necessitated. Accurate diagnosis should await the results by authenticated methods. Quickness is important.

As might be seen from our paper, the need to pretreat urine to compensate for the signals of glycerol is only applicable to glyceroluria, which is very rare. Our method will be used for selected cases for clinical use; it should suffice for clinical screening.

NMR analysis of freeze-dried (and redissolved in DMSO) samples of biological fluid had not been reported. Wilson and Nicholson reported the absence of a signal of urea in the conventional method, but detected it in the freezedried/DMso solution. This phenomenon is well known and is not new.

Both the careful clinician's eye and the quick screening of biological fluid will afford several important pieces of information that one would not be able to get by using only a higher magnetic field NMR spectrometer. Comparisons of the ability of various NMR machines is not a usual goal for researchers, I guess.

Shuichi Yamaguchi

Saitama Children's Med. Ctr. Iwatsuki, Saitama, Japan

\section{Urinary Excretion of Digoxin-like Immunoreactivity after Physical Exerclse}

\section{To the Editor:}

Several studies (for review see refs. 1-4) document the presence of an endogenous substance or group of substances with digoxin-like immunoreactivity (DLIS) in blood of experimental animals and humans, found when RIA or enzyme immunoassay methods are used for assay of digoxin. Experimental studies and theoretical considerations suggest that DLIS might be an endogenous modulator of $\mathrm{Na}^{+} / \mathrm{K}^{+}$. transporting ATPase (EC 3.6.1.37; the receptor of cardiac glycosides) and play a role in the regulation of fluids and electrolytes, as well as in the pathogenesis of several cardiovascular and kidney diseases $(1,3,4)$. In a previous preliminary study (5), DLIS concentrations in serum have also been shown to increase after strenuous physical activity.
Because DLIS concentrations in plasma or serum samples from normal adult subjects are frequently near the limit of sensitivity for RIA methods (68 ), the sample usually must be concentrated before analysis, to increase the precision of the assay. In contrast, urine of adults and newborns have DLIS concentrations four- to sixfold those in plasma, and the direct RIA could be preferable (8).

To investigate whether urinary excretion of DLIS increases during physical activity, concomitantly with the previously reported (5) increase in serum, we measured the urinary excretion rate of DLIS in two groups of athletes, at rest and after a training session.

We measured urinary DLIS by a previously reported solid-phase RIA method $(6,7)$ in which digoxin dissolved in a buffer containing $\mathbf{4 0} \mathrm{g}$ of human serum albumin per liter is used as standard, ${ }^{125}$ I-labeled digoxin as tracer, and solid phase (antibody-coated test tubes) for bound/free separation. Results are expressed as digoxin equivalents.

We directly assayed 0.2-mL samples of urine. The mean sensitivity obtained in 20 separate experiments, performed during nine months, was 2.98 (SD 1.11) pg per tube. The between-assay CV ranged between $10 \%$ and $20 \%$.

We studied nine female volleyball athletes (ages 17-24 y) and 13 male cyclists $(21-37 \mathrm{y})$. The women collected urine samples $2 \mathrm{~h}$ before and just after a 2-h training session (energy expenditure about $200-250 \mathrm{kcal} / \mathrm{h}$ ). The men collected 24-h urine samples (the time of collection was exactly recorded) during a day of training ( $5 \mathrm{~h}$ of training, energy expenditure about 400-450 $\mathrm{kcal} / \mathrm{h}$ ). In addition, we studied a control group of 22 normal subjects (10 men and 12 women, ages $22-55 \mathrm{y}$ ), who also collected 24-h urines during a day of normal activity (the time of collection was also exactly recorded).

The mean daily urinary excretion rate of DLIS by the 22 normal subjects [84.97 (SD 31.35) pg/min] significantly exceeded $(P<0.05)$ that observed in the 13 cyclists [66.92 (SD 17.17) $\mathrm{pg}$ / min]. The mean urinary excretion rate of DLIS found before exercise [88.90 (SD 83.71) $\mathrm{pg} / \mathrm{min}$ ] in the nine volleyball athletes was higher, though not significantly so $(P=0.374$, paired $t$ test), than the value observed after training [64.80 (SD 29.10) pg/min].

Thus we saw no increase in the urinary excretion of DLIS during exercise, even though serum DLIS concentrations reportedly increase after strenuous physical activity (5). Our study suggests that this increase in DLIS in serum after exercise could be at least partly ascribed to a decrease of its removal via the kidney. Or possibly the increase in circulating DLIS is proportional to the intensity of exercise. Further studies in which serum concentration and urinary excretion of DLIS are measured concomitantly after physical exercises of different intensity are necessary to confirm this hypothesis.

\section{References}

1. Valdes Jr R. Endogenous digoxin-like immunoreactive factors; impact on digoxin measurements and potential physiological implications. Clin Chem 1985;31:1525-32.

2. Soldin SJ. Digoxin-issues and controversies [Review]. Clin Chem 1986;32:5-12. 3. Clerico A, Ghione S, Balzan S, Del Chicca MG. Digoxin-like immunoreactive substance: non-specific interference or a new hormone? J Nucl Med Allied Sci 1984;28:297-301.

4. De Wardener HE, Clarkson EM. Concept of natriuretic hormone. Physiol Rev 1985;65:658-759.

5. Valdes Jr R, Hagberg JM. Exercise-induced elevation of endogenous digoxin-like immunoactive factor in human serum [Abstract]. Clin Chem 1985;31:1008.

6. Balzan S, Clerico A, Del Chicca MG, et al. Digoxin-like immunoreactivity in normal human plasma and urine, as detected by a solid-phase radioimmunoassay. Clin Chem 1984;30:450-1.

7. Clerico A, Del Chicca MG, Montreggi A, et al. Digoxin-like immunoreactivity in human body fluids. J Nucl Med Allied Sci 1985;29:305-12.

8. Clerico A, Ghione S, Del Chicca MG, Balzan S. Problems in standardization of digitalis-like substance assays by means of competitive immunological methods [Letter]. Clin Chem 1987;33:340-1.

\section{Aldo Clerico Antonio Cambi Maria Grazia Del Chicca Luigi Cechini Stefano Giaconi}

C.N.R. Instit. of Clin. Physiol. and Postgrad. School of Sport Med.

University of Pisa

56100 Pisa, Italy

\section{Variation among Instruments in Interference by Cephalosporin in the Jaffe Reaction for Creatinine}

\section{To the Editor:}

Cephalosporin antibiotics interfere in the Jaffe reaction for creatinine estimation, and different cephalosporin antibiotics interfere to different extents (1). 